\title{
Energy sharing based on microscopic level densities
}

\author{
Jorgen Randrup ${ }^{1,2, *}$, Martin Albertsson ${ }^{3}$, Gillis Carlsson ${ }^{3}$, Thomas D申ssing ${ }^{4}$, \\ Peter Möller ${ }^{5}$, and Sven Åberg ${ }^{3}$ \\ ${ }^{1}$ Lawrence Berkeley National Laboratory, Berkeley, California 94720, USA \\ ${ }^{2}$ Yukawa Institute for Theoretical Physics, Kyoto 606-8502, Japan \\ ${ }^{3}$ Mathematical Physics, Lund University, S-221 00 Lund, Sweden \\ ${ }^{4}$ Niels Bohr Institute, DK-2100 Copenhagen $\phi$, Denmark \\ ${ }^{5}$ Moller Scientific Graphics and Computing, Los Alamos, New Mexico 87545, USA
}

\begin{abstract}
The transformation of a moderately excited heavy nucleus into two excited fission fragments is modeled as a strongly damped evolution of the nuclear shape. The resulting Brownian motion in the multi-dimensional deformation space is guided by the shape-dependent level density which has been calculated microscopically for each of nearly ten million shapes (given in the three-quadratic-surfaces parametrization) by using a previously developed combinatorial method that employs the same single-particle levels as those used for the calculation of the pairing and shell contributions to the five-dimensional macroscopic-microscopic potential-energy surface.

The stochastic shape evolution is followed until a small critical neck radius is reached, at which point the mass, charge, and shape of the two proto-fragments are extracted. The available excitation energy is divided statistically on the basis of the microscopic level densities associated with the two distorted fragments. Specific fragment structure features may cause the distribution of the energy disvision to deviate significantly from expectations based on a Fermi-gas level density.
\end{abstract}

After their formation at scission, the initially distorted fragments are being accelerated by their mutual Coulomb repulsion as their shapes relax to their equilibrium forms. The associated distortion energy is converted to additional excitation energy in the fully ac- celerated fragments. These subsequently undergo sequential neutron evaporation which is calculated using again the appropriate microscopic level densities. The resulting de- pendence of the mean neutron multiplicity on the fragment mass, $\bar{v}(A)$, as well as the dependence of $\bar{v}(A)$ on the initial excitation energy of the fissioning compound nucleus, exhibit features that are similar to the experimentally observed behavior, suggesting that the microscopic energy sharing mechanism plays an important role in lowenergy fission.

\footnotetext{
*e-mail: JRandrup@LBL.gov
} 


\section{Introduction}

We illustrate here how shape-dependent microscopic level densities may be used to refine the theoret- ical modeling of nuclear fission. Our focus is on fission of moderately excited compound nuclei, such a thermal neutron-induced fission of actinides.

\section{Microscopic shape-dependent level density}

In Ref. [1] a method was developed for microscopic calculations of level densities for deformed nuclei and it has been adapted to the fission process [2]. For a specified shape $\chi$, the single-particle levels for protons and neutrons needed for the combinatorial calculation of the level density are obtained by solving the Schrödinger equation in the associated foldedYukawa potential. These are the same levels as those previously used in Ref. [3] to calculate the microscopic shell and pairing energies in the construction of the deformation energy surface, thus guaranteeing consistency of the treatment. The corresponding local many-body vacuum state $|0 ; \chi\rangle$ has $N$ neutrons and $Z$ protons filling the lowest single-particle states and the uncorrelated excited states consist of all multiple particle-hole excitations,

$$
|i ; \chi\rangle=\prod_{n \geq 1} a_{v_{n}^{(i)}}^{\dagger} a_{\mu^{(i)}}|0 ; \chi\rangle
$$

For each many-body state $|i ; \chi\rangle$, blocked BCS calculations for neutrons and protons separately provide the state-dependent pairing gaps, $\Delta_{i}^{n}(\chi)$ and $\Delta_{i}^{\mathrm{p}}(\chi)$, respectively, and the energy of the correlated intrinsic many-body state, $E_{i}(\chi)=E_{i}^{n}(\chi)+E_{i}^{\mathrm{p}}(\chi)$.
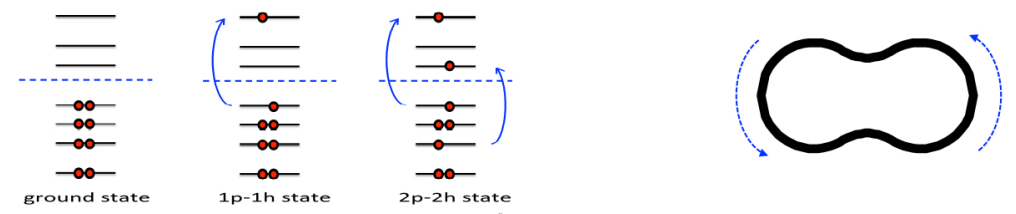

Figure 1. Illustration of the calculation of the microscopic level density for a specified shape, $\rho(E, I, \chi)$. For each many-particle-many-hole excitation, a BCS pairing calculculation is carried out for neutrons and protons separately, yielding the associated correlated energy of the intrinsic state, $E_{i}^{n}(\chi)+E_{i}^{\mathrm{p}}(\chi)$ (left panel); each such intrinsic state forms the head of a rotational band contributing a rotational energy, $E_{i}^{\text {rot }}(I ; \chi)($ right panel).

For each such intrinsic state, the angular momentum along the nuclear symmetry axis is denoted by $K$ and it is assume that it forms a rotational band head. The resulting total angular momenta $I$ may then take on the values $I=K,(K+1),(K+2), \ldots$ and the corresponding rotational energies of the band members are

$$
E_{i}^{\mathrm{rot}}(I ; \chi)=\left[I(I+1)-K_{i}(\chi)^{2}\right] /\left[2 \mathcal{J}_{\perp}\left(\chi, \Delta_{i}^{\mathrm{n}}(\chi), \Delta_{i}^{\mathrm{p}}(\chi)\right)\right]
$$

The moment of inertia $\zeta_{\perp}$ is approximated by the moment of inertia of a rigid body with the shape $\chi$, modified by the calculated pairing gaps for the state [4].

In Ref. [1] it was found that the enhancements arising from collective vibrational modes are unim- portant and they are therefore ignored in the present fission applications. The total energy of a state is then given by

$$
E_{i}(I, \chi)=E_{i}^{\mathrm{n}}(\chi)+E_{i}^{\mathrm{p}}(\chi)+E_{i}^{\mathrm{rot}}(I ; \chi) .
$$


In terms of these energies, the level density is obtained by binning,

$$
\rho(E, I, \chi)=\frac{1}{\Delta E} \int_{E}^{E+\Delta E} \sum_{i} \delta\left(E^{\prime}-E_{i}(I, \chi)\right) d E^{\prime} .
$$

Thus, for each shape $\chi$, the resulting states are binned according to their energy $E_{i}$ and their total angular momentum $I$; the bin width was taken as $\Delta E=200 \mathrm{keV}$. The sensitivity of the results to the bin width has been tested and it was found that a doubling or tripling of $\Delta \mathrm{E}$ has no discernable effect on the calculated observables.

\section{Brownian shape evolution}

The description of nuclear fission as a generalized Brownian motion builds on the assumption that the evolving nucleus can be characterized by its shape degrees of freedom $\chi$. The associated shape param- eters are treated as classical variables that are coupled dissipatively to the remaining nuclear degrees of freedom which have a primarily microscopic character and are assumed to have a short relaxation time. The resulting large-amplitude collective motion then exhibits a strongly damped diffusive evo- lution that can be described as a random walk on the associated multi-dimensional potential-energy surface $U(\chi)$. When the values of the potential are available on a discrete lattice of shapes (as is the case for the 5D tabulations made in Ref. [5]), the Smoluchowski equation can be approximately simulated by means of a random walk on this lattice [6].

The rates of transition between two lattice sites $\chi$ and $\chi^{\prime}, v\left(\chi \leftrightarrow \chi^{\prime}\right)$, and the corresponding local level densities, $\rho(\chi)$ and $\rho\left(\chi^{\prime}\right)$, must satisfy detailed balance, $\rho(\chi \rightarrow) v\left(\chi \rightarrow \chi^{\prime}\right)=$ $\rho\left(\chi^{\prime}\right) v\left(\chi^{\prime} \rightarrow \chi\right)$. A particularly simple way of ensuring dertailed balance is presented by the Metropolis procedure [7] according to which a proposed shape change from $\chi$ to $\chi^{\prime}$ is accepted unconditionally if $\rho\left(\chi^{\prime}\right)>\rho(\chi)$ (“downhill”), whereas it is accepted only with the probabilty $\rho\left(\chi^{\prime}\right) / \rho(\chi)$ otherwise ("uphill”).

In Ref. [6] and all subsequent appli,cations until recently [2], a simplified Fermi-gas leveldensity expression was used, $\bar{\rho}_{E}(\chi) \approx \exp \left[2 \sqrt{a_{A}(E-U(\chi))}\right]$ with $a_{A} \approx A /(10 \mathrm{MeV})$. Because the level density then depends on the shape $\chi$ only through the local excitation energy $E^{*}(\chi)$ $=E-U(\chi)$, the ratio between neighboring level densities can be approximated by means of a logarithmic expansion,

$$
\frac{\tilde{\rho}\left(\chi^{\prime}\right)}{\tilde{\rho}(\chi)} \approx \exp \left[-\frac{\partial \ln \tilde{\rho}\left(E^{*}\right)}{\partial E^{*}} \frac{\partial U(\chi)}{\partial \chi} \Delta \chi\right] \approx \mathrm{e}^{-\Delta U / T},
$$

where $\Delta U=U\left(\chi^{\prime}\right)-U(\chi)$ is the change in the potential energy associated with the proposed shape change $\chi \rightarrow \chi^{\prime}=\chi+\Delta \chi$ and $T(\chi)=1 /\left[\partial \ln \tilde{\rho}\left(E^{*}\right) / \partial E^{*}\right]$ is the local nuclear temperature. In the present treatment, we follow Ref. [2] and employ the microscopic level densities (see Sect. 2) to evaluate the required ratios $\rho\left(\chi^{\prime}\right) / \rho(\chi)$. The use of microscopic level densities is particularly well- suited for describing the dependence of the shape evolution on the total excitation energy of the fissioning system because the microscopic pairing and shell effects automatically subside as the energy is raised, without the need for any adjustable parameters.

\section{Scission}

We focus here on fission of excited ${ }^{236} \mathrm{U}$. An ensemble of scission configurations is generated by performing one million Metropolis walks, starting from well inside the outer fission barrier 


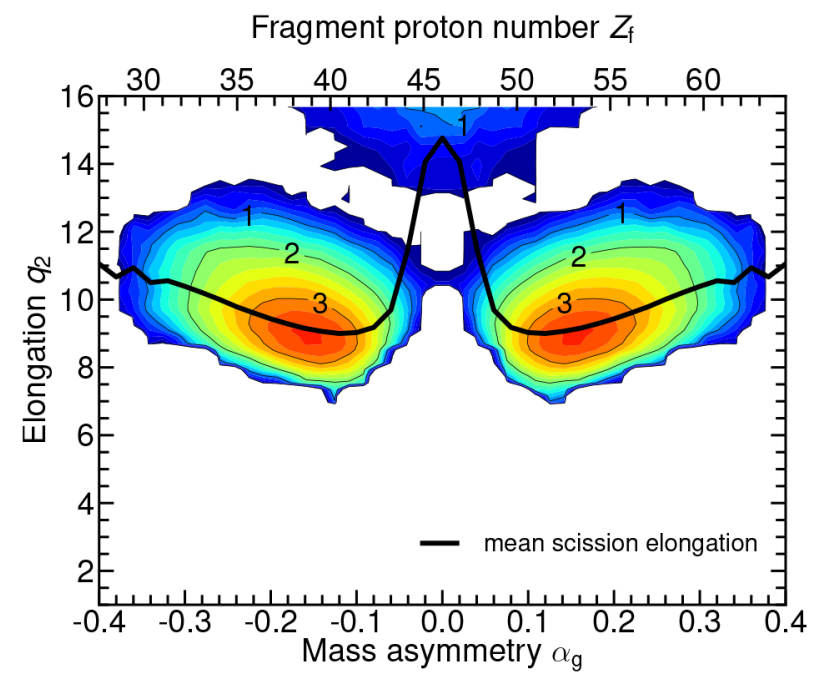

Figure 2. Logarithmic contour plot of the location of the end shapes projected onto the plane of mass asymmetry $\alpha_{\mathrm{g}}$ (corresponding the lattice index $M=-33: 33$ ) and relative quadrupole moment $q_{2}$ (corresponding the lattice index $I=1: 45$ for ${ }^{235} \mathrm{U}\left(\mathrm{n}_{\mathrm{th}}, \mathrm{f}\right)$; the fragment charge numbers $Z_{\mathrm{f}}=46\left(1+\alpha_{\mathrm{g}}\right)$ are shown on the upper axis (from Ref. [2]). The distribution has a dominant asymmetric component and a relatively small symmetric component at significantly larger elongations. Because the lattice employed in [2] does not extend sufficiently far in $q_{2}$ to fully encompass the symmetric component it has been extended upwards to $I=53$ for the present study.

and continuing until the neck has shrunk to a specified critical value, $c_{0}=1.5 \mathrm{fm}$. Figure 2 illustrates the distribution of these end shapes.

Each end shape is assumed to be abruptly transformed into two proto-fission fragments whose shapes are obtained by completing the two spheroidal shape segements, as illustrated in Fig. 3. (An overall rescaling is subsequently performed to conserve the total volume.)

The proto-fragments are generally distorted relative to their equilibrium shapes to which they relax as they move apart due to their mutual Coulomb repulsion. The associated distortion energies,

$$
E_{i}^{\mathrm{dist}} \approx M_{i}\left(\varepsilon^{\mathrm{sc}}\right)-M_{i}\left(\varepsilon^{\mathrm{gs}}\right), i=\mathrm{H}, \mathrm{L},
$$

are assumed to be converted into additioanl statistical excitation energy in the respective fragments before the neutron evaporation commences.

Because the collective kinetic energy associated with the shape evolution is assumed to be neg- ligible prior to scission, we take the available excitation energy at scission as the difference between the total energy, $E$, and the potential energy of the end configuration,

$$
E_{\mathrm{sc}}^{*}=E-U\left(q_{2}^{\mathrm{sc}}, c^{\mathrm{sc}}, \varepsilon_{1}^{\mathrm{sc}}, \varepsilon_{2}^{\mathrm{sc}}, \alpha_{\mathrm{g}}^{\mathrm{sc}}\right) .
$$

The key assumption in the present study is that this energy is divided among the two protofragments statistically based on the microscopic level densities associated with their distorted shapes. Thus the excitation energy in the heavy fragment is governed by the following probability distribution,

$$
P\left(E_{\mathrm{H}}^{*} ; E_{\mathrm{sc}}^{*}\right) \sim \rho_{\mathrm{H}}\left(E_{\mathrm{H}}^{*} ; \varepsilon_{\mathrm{H}}^{\mathrm{sc}}\right) \rho_{\mathrm{L}}\left(E_{\mathrm{sc}}^{*}-E_{\mathrm{H}}^{*} ; \varepsilon_{\mathrm{L}}^{\mathrm{sc}}\right)
$$




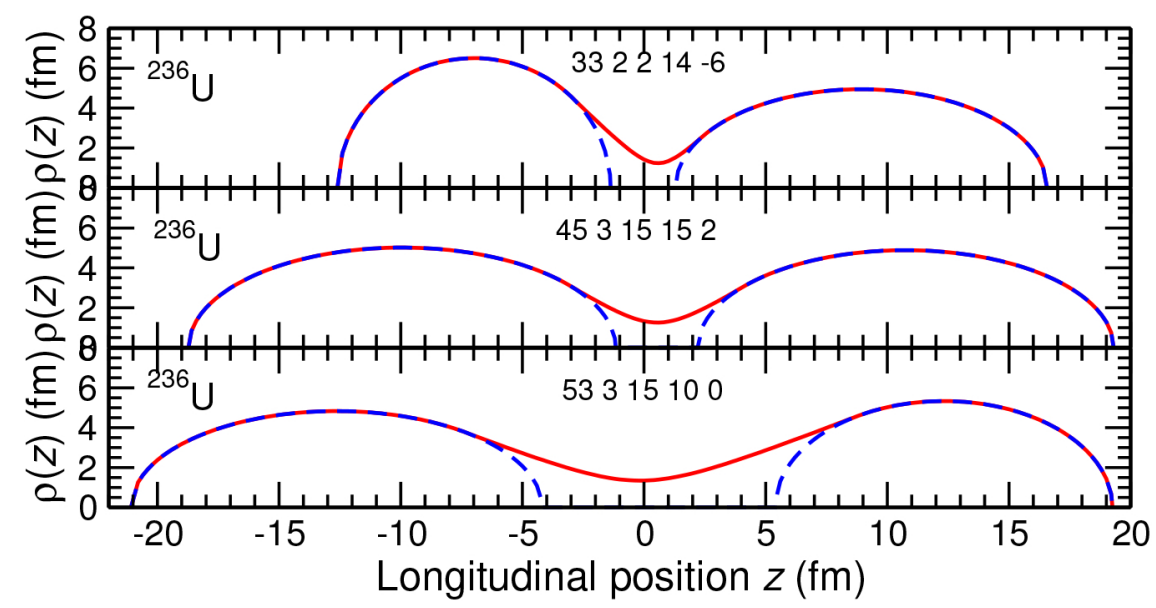

Figure 3. Three examples of the scission procedure: For each end shape (characterized by having a neck radius $c$ equal to the specified critical value, $c_{0}=1.5 \mathrm{fm}$ ), each of the two spheroidal endcaps of the $3 \mathrm{QS}$ shape is completed and the resulting two-spheroid shape is renormalized to conserve volume. The top panel shows an asymmetric end shape (which is fairly compact), whereas the two lower panels show end shapes that are nearly mass symmetric (and rather elongated). The shape lattice indices ( $I, J$, $K, L, M)$ are indicated for each shape.

Figure 4 shows the energy distribution $P\left(E_{\mathrm{H}} ; E_{\mathrm{sc}}^{*}\right)$ at three different values of the total available energy $E_{\mathrm{sc}}^{*}$ for two different mass divisions having $\left(A_{\mathrm{H}}: A_{\mathrm{L}}\right)=(130: 106)$ and $(152: 84)$. These two divisions contribute to the yields at the inner and outer wings of the double-humped mass distribution, respectively, and the fragment deformations considered are typical of those divisions.

For the case shown in the top panel of Fig. 4, the heavy fragment, ${ }^{130} \mathrm{Sn}$, is very close to being doubly magic and it therefore has a spherical ground-state shape, $\varepsilon_{\mathrm{H}}^{\mathrm{gs}}=0$, while the light partner fragment, ${ }^{106} \mathrm{Mo}$, has a well-deformed prolate ground-state shape, $\varepsilon_{\mathrm{L}}^{\mathrm{gs}}=0.33$. The fragment deformations at scission are $\varepsilon_{\mathrm{H}}^{\mathrm{sc}}=-0.10$ and $\varepsilon_{\mathrm{L}}^{\mathrm{sc}}=0.30$ which both deviate only slightly from the respective ground-state deformations. The near magicity of the heavy fragment (with a shell correction energy of $-10.2 \mathrm{MeV}$ ) causes the level density to remain very small up to excitation energies of $20 \mathrm{MeV}$. Conversely, the shell correction energy of the light fragment is $+0.35 \mathrm{MeV}$ and its level density is considerably larger than that of the heavy partner in that energy range. As a consequence, the major part of the energy goes to the light fragment, the most probable division being about $2: 8 \mathrm{MeV}$. This is very different from the Fermi-gas scenario in which the most probable division is $5.5: 4.5 \mathrm{MeV}$.

The opposite appears when the two fragments differ more in size, as illustrated in the bottom panel of Fig. 4. Here the microscopic energy-partition distribution function strongly favors the heavy fragment, ${ }^{152} \mathrm{Nd}$, relative to the light fragment, ${ }^{84} \mathrm{Ge}$. In this case, the typical scission deformation of the heavy fragment is considerably smaller, $\varepsilon_{\mathrm{H}}^{\mathrm{sc}}=0.10$, than its ground-state deformation, $\varepsilon_{\mathrm{H}}^{\mathrm{gs}}=0.24$. Therefore the heavy fragment has a large single-particle level density and, consequently, it has a large positive shell correction, $\approx+6.1 \mathrm{MeV}$ (as compared to $\approx 6.9 \mathrm{MeV}$ for the ground-state shape) and a particularly high level density. On the other hand, the light fragment, ${ }^{84} \mathrm{Ge}$, has a neutron number that is close to being magic, $N_{\mathrm{L}}$ $=52$, so its level density is low. As a result, the heavy fragment is strongly favored in the energy division, even up to quite high energies, as clearly seen in Fig. 4 (d)-(f). 


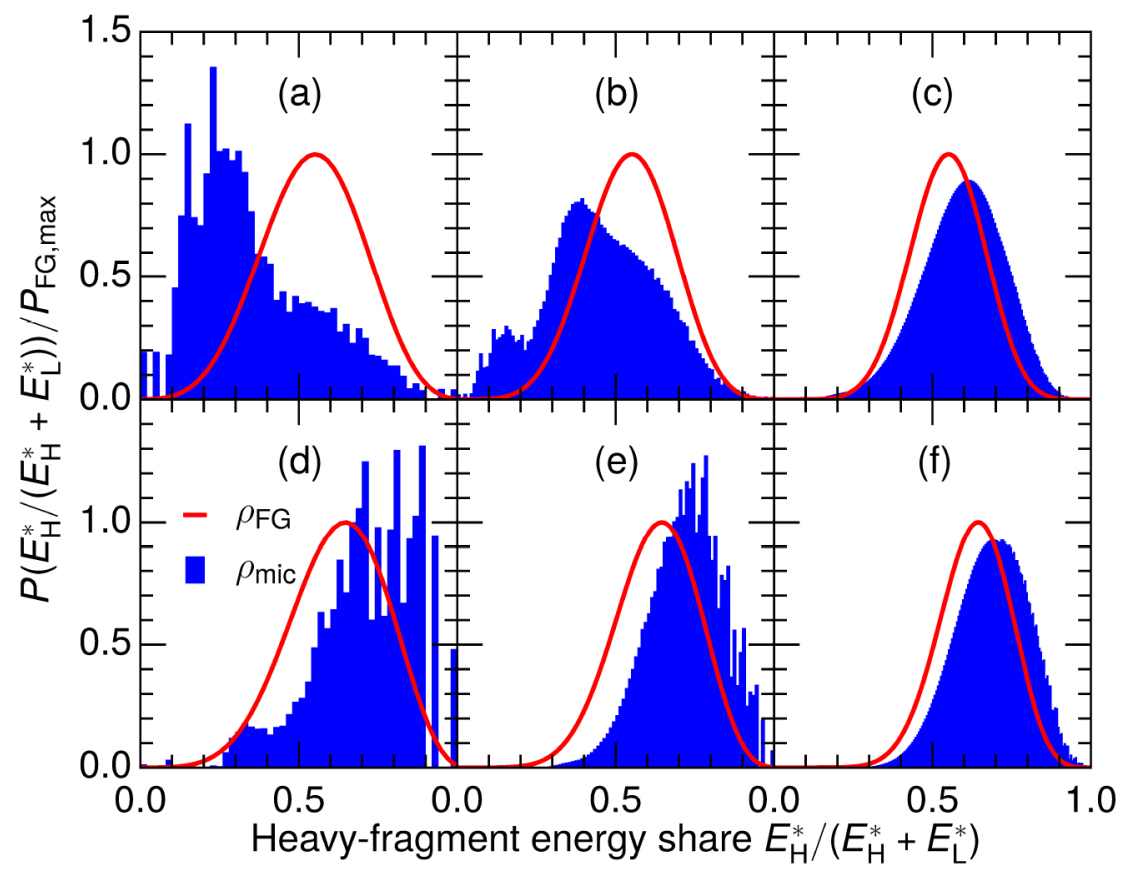

Figure 4. For ${ }^{235} \mathrm{U}(\mathrm{n}, \mathrm{f})$ is shown the distribution function $P\left(E_{\mathrm{H}}^{*} ; E_{\mathrm{sc}}^{*}\right)$ giving the probability that the heavy primary fission fragment acquires the excitation energy $E_{\mathrm{H}}^{*}$ when the total excitation energy available at scission is $E_{\mathrm{sc}}^{*}$, for two different divisions, either 130+106: $(N, Z, \varepsilon)_{\mathrm{H}}=(80,50,-0.1)$ and $(N, Z, \varepsilon)_{\mathrm{L}}=(64,42,+0.3)$ (top panels) or 152+84: $(N, Z, \varepsilon)_{\mathrm{H}}=(92,60,+0.1)$ and $(N, Z, \varepsilon)_{\mathrm{L}}=(52,32$, $+0.1)$ (bottom panels), and three different values of the total available energy at scission: $E_{\mathrm{sc}}^{*}=10$ (left column), 20 (center column), 40 (right column) MeV. In each panel, the distributions obtained from the shape-dependent microscopic level densities $\rho(E ; \chi)$ (blue histograms) and those obtained with the simplified Fermi-gas level density $\rho_{\mathrm{FG}}\left(E^{*}\right)$ (red curves) have been scaled with a common factor so that the maximum value of the Fermi-gas result is unity.

As the available energy $E_{\mathrm{sc}}^{*}$ is increased, the microscopic energy partition distribution, $P\left(E_{\mathrm{H}}^{*} ; E_{\mathrm{sc}}^{*}\right)$, approaches the macroscopic form obtained with the simplified Fermi-gas level density, $\tilde{\rho}_{E}(\chi)$, and the structure effects in the energy partition distribution gradually subside.

For each scission configuration obtained at the end of the Metropolis walk, the excitation ener- gies of the proto-fragments are sampled from the appropriate microscopic partition distribution (8) illustrated in Fig. 4. Figure 5 shows the mean excitation and distortion energies, $\bar{E}_{\mathrm{sc}}(A)$ and $\bar{E}_{\mathrm{dist}}(A)$, together with their sum, the resulting total excitation energy of the primary fission fragment.

The mean fragment excitation energy at scission has a pronounced structure that may be qual- itatively understood from the energy partition distribution functions of the two examples discussed above. The local minimum slightly below $A \approx 130$ and the local maximum around $\approx 106$ result from the favoring of the light fragment in the energy sharing, illustrated in Fig. 4 (a) - (c), while the pronounced maximum at $A \approx 150$ and the relatively low values in the $A \approx 84$ region result from the favoring of the heavy fragment, illustrated in Fig. 4 (d) - (f).

Due to overall energy conservation, the energetics of the fragments is coupled to their kinetic en- ergies (which are not discussed here due to space limitations). For the asymmetric fission component (see Fig. 2), the mass dependence of the total fragment kinetic energies, 


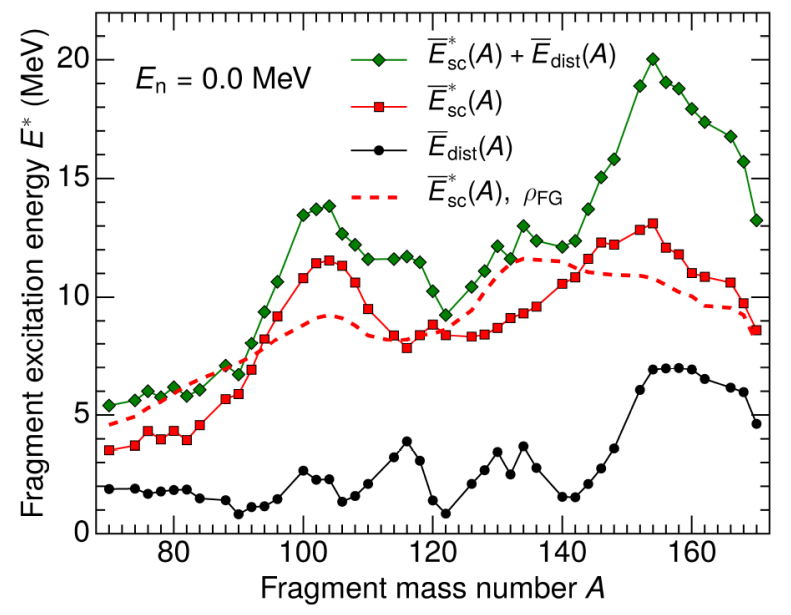

Figure 5. As functions of the fragment mass number $A$ are shown the mean fragment distortion energy $E_{\text {dist }}(A)$ (black dots), the mean excitation energy at scission, $E_{\mathrm{sc}}^{*}(A)$ (red squares), and the sum, $E_{\mathrm{dist}}(A)$ $+E_{\mathrm{sc}}^{*}(A)$ (green diamonds). as extracted from an ensemble of $10^{6}$ scission configurations. For reference is also shown the value of $E_{\mathrm{sc}}^{*}(A)$ that would be obtained with the simple Fermi-gas level density $\rho_{\mathrm{FG}}\left(E^{*}\right)$ (red dashes).

$\operatorname{TKE}(A)$, generally agrees very well with the experimental data. But for the symmetric component TKE comes out too high which leads to too low values of $E_{\mathrm{sc}}^{*}$. This problem is currently under investigation.

After a fragment has been fully accelerated and its shape has relaxed to its ground-state form, it disposes of its excitation energy by neutron evaporation (see Appendix A) and, on a longer time scale, by radiation of photons. The dependence of the mean neutron multiplicity on the fragment mass, $\bar{v}(A)$, may be used to test the calculated energy partitioning.

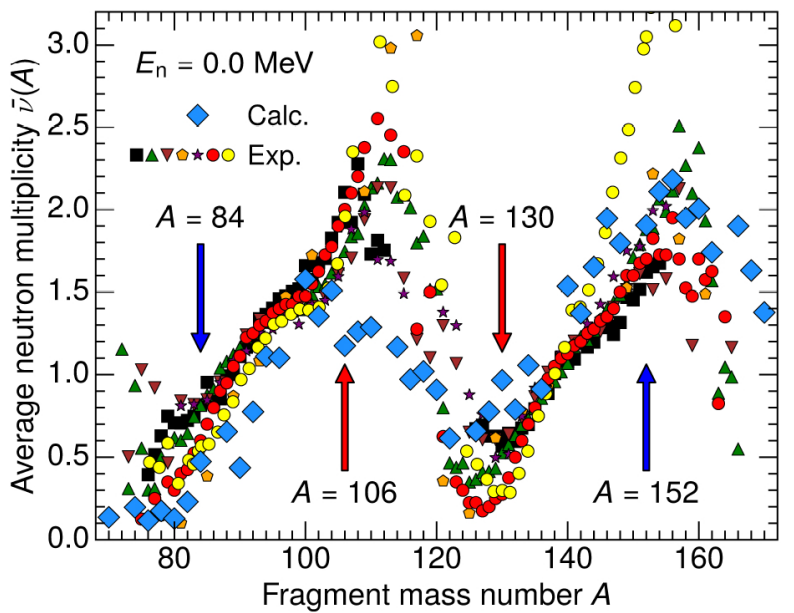

Figure 6. The mean neutron multiplicity, $\bar{v}$, as a function of the mass number of the primary fission fragment, $A$, for ${ }^{235} \mathrm{U}\left(\mathrm{n}_{\mathrm{th}}, \mathrm{f}\right)$. The calculated results (blue diamonds) are shown together with available experimental data: Nishio et al. [8] (black squares), Apalin et al. [9] (yellow circles), Vorobyev et al. [10] (green triangles), Batenkov et al. [11] (orange diamonds), Boldeman et al. [12] (purple stars), Maslin et al. [13] (brown triangles), Göök et al. [14] (red circles). The red/blue arrows point to the mass divisions examined in Fig. 4.

Figure 6 shows the calculated mean neutron multiplicity $\bar{v}(A)$ together with data from a variety of experiments. The sawtooth appearance of the data is reasonably well reproduced by the calculation. It arises from a combined effect of the behavior of the neutron separation energy, $S_{\mathrm{n}}(A)$, which displays a jump near $A=132$ due to the closed shells at $Z=50$ and $N$ 
$=82$, and the behavior of the total fragment excitation energy $E_{\mathrm{sc}}^{*}(A)+E_{\mathrm{dist}}^{*}(A)$ displayed in Fig. 5. The significant shortfall of $\bar{v}(A)$ in the symmetric region arises in large part from the fact that the employed 3 QS shape family is somewhat inadquate for the very elongated scission configurations of the mass-symmetric component (which also leads to too high fragment kinetic energies for near-symmetric divisions).

We have also studied how $\bar{v}(A)$ changes as the excitation energy of the fissioning nucleus is in- creased. The sawtooth behaviour is then weakened due to the reduction of the shell corrections near $A=130$ which significantly increases the level density in the heavy fragment and hence $\bar{v}_{\mathrm{H}}$. Such an evolution is also seen in the experimental data. The calculated $\bar{v}(A)$ gradually approaches the form obtained if the excitation energy were divided according to the masses, as suggested by the simplified Fermi-gas level density.

\section{Conclusion}

In order to elucidate how the excitation energy is divided between the two fission fragments, we have augmented the recently developed level-density guided Metropolis shape evolution treatment [2] with shape-dependent microscopic level densities for the proto-fragments which are distorted relative to their equilibrium shapes. The available energy is partitioned statistically according to the corresponding microscopic level densities which take account of the structure effects in the proto-fragments. After separation, the distortion energy is converted into additional excitation before the sequential neutron evaporation commences. The dependence of the resulting mean neutron multiplicity on the mass number of the primary fragment agrees reasonably well with the data. In particular, the sawtooth appearance can be understood to result in part from shell-structure effects in the level densities as well as from structure in the multi-dimensional potential-energy surface.

This report is based in part on the collaborative work reported in Ref. [16]. It was supported by the Swedish Natural Science Research Council (BGC and SA) and by the Office of Nuclear Physics in the U.S. Department of Energy's Office of Science under Contract No. DE-AC02-05CH11231 (JR).

\section{Appendix A Neutron evaporation}

The fragment-mass dependence of the average neutron multiplicity, $\bar{v}$, presents a convenient observable that is sensitive to the division of the excitation energy bewteen the primary fragments. We have therefore simulated the evaporation cascades using the simple Weisskopf-Ewing treatment in which the neutron spectrum is assumed to be of the form, $d N / d \varepsilon \sim \sqrt{\varepsilon} \rho\left(E_{\max }^{*}-\varepsilon\right) \sqrt{\varepsilon d \varepsilon}$. The last factor comes from the neutron phase space $d^{3} p$ and the level density refers to the daughter at the excitation $E_{\max }^{*}-\varepsilon$ where $E_{\max }^{*}=E^{*}-S_{\mathrm{n}}$ is the maximum possible excitation energy in that fragment after the evaporation (corresponding to the emission of a neutron with vanishing kinetic energy, $\varepsilon=0$ ); $E^{*}$ is the excitation energy of the mother fragment and $S_{\mathrm{n}}$ is its neutron separation energy. The first factor represents the transmission coefficient which is assumed to be proportional to the transverse speed of the neutron. In the present work, we use the microscopic level density for the evaporation daughter nucleus, evaluated for its ground-state shape $\varepsilon_{\mathrm{gs}}$, and we repeat the evaporation procedure as long as it is energetically posisble to emit a neutron, i.e. as long as $E^{*}$ exceeds $S_{\mathrm{n}}$, because the competiton from photon emission has an insignificant effect on $\bar{v}$.

Often an analytical form of the level density is employed (for example, in FREYA, a simplified Fermi-gas from is used, $\left.\rho\left(E^{*}\right) \sim \exp \left(2 \sqrt{a_{A} E^{*}}\right)\right)$. Then $\ln \rho\left(E^{*}\right)$ may be expanded around $E^{*}=E_{\text {max }}^{*}$,

$$
\ln \rho\left(E_{\max }^{*}-\varepsilon\right) \approx \ln \rho\left(E_{\max }^{*}\right)-\varepsilon\left[d \ln \rho\left(E^{*}\right) / d E^{*}\right]_{E^{*}=E_{\max }^{*}}=\ln \rho\left(E_{\max }^{*}\right)-\varepsilon / T_{\max },
$$


where $T_{\max }$ is the maximum temperature in the daughter, leading to $d N / d \varepsilon \sim \varepsilon \exp \left(-\varepsilon / T_{\max }\right)$ $d_{\varepsilon}$. For the present explorative study, this simple treatment would suffice.

\section{References}

[1] H. Uhrenholt, S. Åberg, A. Dobrowolski, T. Dфssing, T. Ichikawa, and P. Möller, Nucl. Phys. A 913, 127 (2013)

[2] D. Ward, B. G. Carlsson, T. Dфssing, P. Möller, J. Randrup, and S. Åberg, Phys. Rev. C 95, 024618 (2017)

[3] P. Möller and J. Randrup, Phys. Rev. C 91, 044316 (2015)

[4] R. Bengtsson and S. Åberg, Phys. Lett. B 172, 277 (1986)

[5] P. Möller, A.J. Sierk, T. Ichikawa, A. Iwamoto, R. Bengtsson, H. Uhrenholt, and S. Åberg, Phys. Rev. C 79, 064304 (2009)

[6] J. Randrup and P. Möller, Phys. Rev. Lett. 106, 132503 (2011)

[7] N. Metropolis, A.W. Rosenbluth, M.N. Rosenbluth, A.H. Teller, and E. Teller, J. Chem. Phys. 21, 1087 (1953)

[8] K. Nishio Y. Nakagome, H. Yamamoto, and I. Kimura, Nucl. Phys. A 632, 540 (1998)

[9] V.F. Apalin, Y. N. Gritsyuk, I.E. Kutikov, V.I. Lebedev, and L. A. Mikaelian, Nucl. Phys. A 71, 553 (1965)

[10] A.S. Vorobyev et al., EPJ Web Conf. 8, 3004 (2010)

[11] O.I. Batenkov et al., AIP Conf. Proc. 769, 625 (2005)

[12] J.W. Boldeman, A.R. de L. Musgrove, and R. L. Walsh, Aust. J. Phys. 24, 821 (1971)

[13] E.E. Maslin, A.L. Rodgers, and W.G.F. Core, Phys. Rev. 164, 1520 (1967)

[14] A. Göök, F.J. Hambsch, and S. Oberstedt, EPJ Web Conf. 169, 4 (2018)

[15] R. Müller, A.A. Naqvi, F. Käppeler, and F. Dickmann, Phys. Rev. C 29, 885 (1984)

[16] M. Albertsson, B.G. Carlsson, T. Dфssing, P. Möller, J. Randrup, S. Åberg, arXiv:1811.02283 